\title{
Particle detection on flat surfaces
}

Jacques van der Donck ${ }^{1}$, Rob Snel, Jetske Stortelder, Alfred Abutan, Sjoerd Oostrom, Sander van Reek, Bert van der Zwan, Peter van der Walle (TNO)

\begin{abstract}
Since 2006 EUV Lithographic tools have been available for testing purposes giving a boost to the development of fab infrastructure for EUV masks. The absence of a pellicle makes the EUV reticles extremely vulnerable to particles. Therefore, the fab infrastructure for masks must meet very strict particle requirements. It is expected that all new equipment must be qualified on particles before it can be put into operation. This qualification requirement increases the need for a low cost method for particle detection on mask substrates.
\end{abstract}

TNO developed its fourth generation particle scanner, the Rapid Nano. This scanner is capable of detecting nanometer sized particles on flat surfaces. The particle detection is based on dark field imaging techniques and fast image processing. The tool was designed for detection of a single added particle in a handling experiment over a reticle sized substrate. Therefore, the Rapid Nano is very suitable for the validation of particle cleanliness of equipment. During the measurement, the substrate is protected against particle contamination by placing it in a protective environment. This environment shields the substrate from all possible contamination source in the Nano Rapid (stages, elevator, cabling). The imaging takes place through a window in the protective cover. The geometry of the protective environment enables large flexibility in substrate shape and size. Particles can be detected on substrates varying from $152 \times 152 \mathrm{~mm}$ mask substrates to wafers up to $200 \mathrm{~mm}$. PSL particles of $50 \mathrm{~nm}$ were detected with signal noise ratio of 26. Larger particles had higher signal noise ratios. By individually linking particles in two measurements the addition of particles can be detected. These results show that the Rapid Nano is capable of detecting particles of $50 \mathrm{~nm}$ and larger of a full reticle substrate.

Key words: EUV reticles, reticle inspection, particle detection, Rapid Nano, qualification

\section{INTRODUCTION}

The second generation EUV lithographic tools are available as yet, propelling the need for fab infrastructure that meets the challenging EUV requirements. For a most issues this change is a gradual one, comparable to the introduction of every new node. An important exception is the way reticles are handled. Since no suitable pellicle material exists, EUV reticles are much more vulnerable to particles ${ }^{[1]}$. In the $193 \mathrm{~nm}$ node, the pellicle protected the reticle against particles smaller than $3 \mu \mathrm{m}$. Particles larger than $20 \mathrm{~nm}$ on the front side ${ }^{[2]}$ and of $100 \mathrm{~nm}$ on the back side may already hamper the functionality of EUV reticles and reduce yield to an unacceptable level.

The handling chain for EUV reticles has gained a lot of attention. All new equipment for the handling chain of EUV reticles must be adapted to this technology. The Dual Pod carrier concept has been introduced in order to minimize contamination effects. New handling equipment should also be compliant with the much more severe particle cleanliness levels required for EUV. Therefore, the new handling equipment must be at least a factor 100 cleaner than in previous nodes. This requirement needs a lot of effort during equipment development to ensure that it can be met over the full tool life time. The high costs of EUV reticles will require that only equipment that can ensure the highest cleanliness level will be used. Therefore, qualification of all equipment that is used in the complete handling chain of EUV reticles is a necessity.

The most reliable method of measuring the cleanliness level within equipment is by processing reticle substrates in the newly developed equipment and measuring the amount of added particles. The availability of particle detection equipment for particles on reticles is a prerequisite for this task. Since transport is still a risk ${ }^{[3]}$ qualification should mainly take place at the equipment suppliers. Therefore, the need for a low cost particle detection tool is expected.

${ }^{1}$ TNO, P.O. Box 155, 2600 AD, Delft, The Netherlands, email: jacques.vanderdonck@tno.nl

Extreme Ultraviolet (EUV) Lithography II, edited by Bruno M. La Fontaine, Patrick P. Naulleau, Proc. of SPIE Vol. 7969, 79691S · @ 2011 SPIE · CCC code: 0277-786X/11/\$18 - doi: 10.1117/12.879435 
Experiments on cleanliness, during development or qualification of reticle handling equipment, are in principle related to determining the number of particles on a substrate surface, performing a number of handling cycles and determining particles on the substrate again.

Subtracting the number of particles observed in the two measurements will lead to errors. Since the measuring tool is used for very clean equipment, the number of observed particles in a qualification test will be very low. Therefore, even the high capture rate of currently available particle detection systems does not give the required precision. A reliable determination of added particles can only be made when each particle is identified, labeled and individually compared. To overcome the errors of false positives and negatives, the raw data are necessary for review and reevaluation. By storing our raw data all times, our approach differs from other particle detection equipment ${ }^{[4]}$.

Additional requirements to reticle inspection tools are that the particle detection equipment should be able to detect one added particle $(>20 \mathrm{~nm})$ on a reticle substrate $(152 \times 152 \mathrm{~mm})$ with a high reliability. Furthermore, it should be able to carry out the measurement in a reasonable time. For routine measurements at a substrate producer or in a mask shop, the measurement time should be relatively short. For development and qualification the number of measurements will be much lower and a longer measurement time can be allowed.

In qualification procedures only number and size of added particles is important. For equipment development it is also important to identify particle materials and deposition patterns. This information enables equipment developers to track particle sources and change the equipment design to eliminate the sources. Material identification of particles is typically performed in a SEM equipped with EDX (energy dispersive X-ray analysis). Time effective SEM-EDX analysis of particles is only possible if particle locations are provided with sufficient accuracy and are reported in a standardized format (e.g. KLA Results Format).

\section{RAPID NANO: THE TNO PARTICLE SCANNER}

TNO has developed its fourth generation particle detection equipment, the Rapid Nano. The tool works on a dark field imaging technology ${ }^{[5]}$. A substrate is illuminated under an angle by a $5 \mathrm{~W}, 532 \mathrm{~nm} \mathrm{ND}$ :YAG laser. The illuminated area is monitored by an ultra sensitive Peltier cooled science camera on an optical column with high quality imaging optics. Polarizers are used to minimize reflections. The substrate moves in a stepping mode underneath the imaging system and after each step an image is acquired. The stage system delivers an accurate position related trigger signal to the camera which ensures the required position accuracy level. The substrate is kept in focus by an interferometry based level sensor and a fast and accurate $\mathrm{z}$-stage. The substrate is protected against additional particle deposition during the measurement by a protective environment, the scan box ${ }^{[6]}$. Imaging is performed through a thin polymeric window (see Figure 1, right). Raw images are stored and processed, enabling reprocessing of data at different sensitivity levels and providing the dark field images of the observed defects. Immediate defect review in live dark field mode is possible and by comparing images of the same locations in two different measurements (e.g. before and after a cycle test in handling equipment) each defect can be checked separately. Particles with sizes around the detection limit can be checked visually off line. Storage of raw data guarantees full traceability of results in length of time.
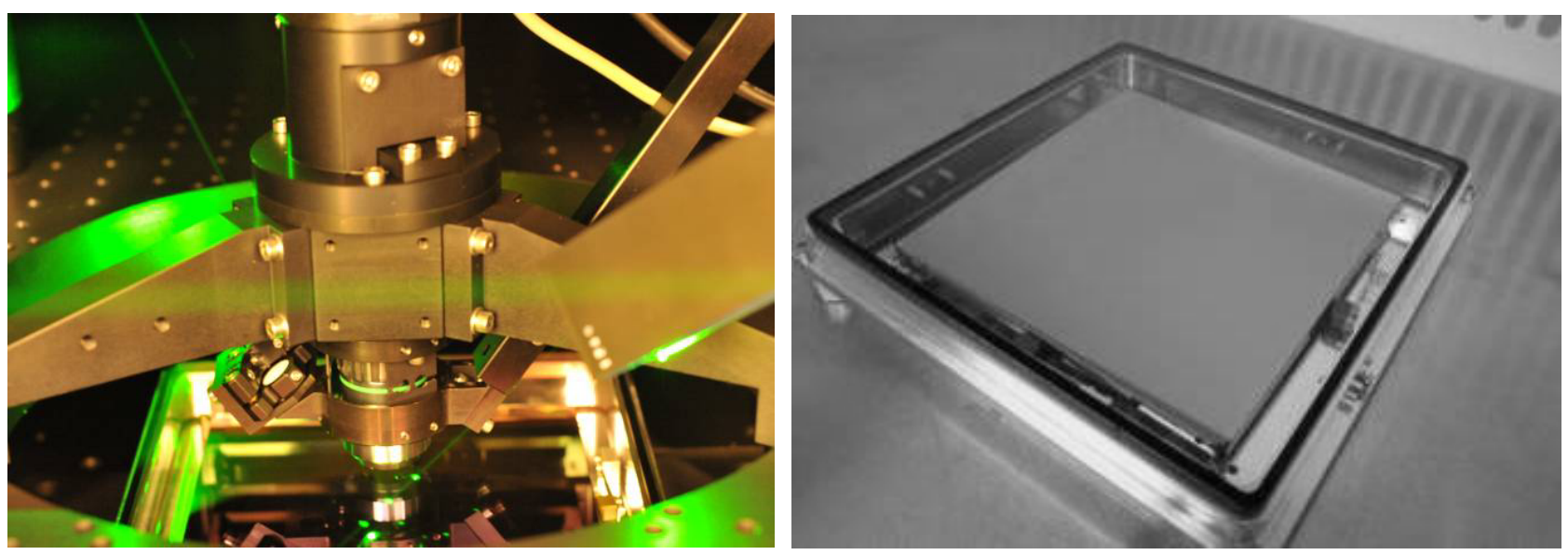

Figure 1: Rapid Nano interior (left), protective environment scan box with window (right) 


\section{PARTICLE DETECTION AND DATA PROCESSING}

Data consist of images in uncompressed TIF format. The homogeneity of the dark field images strongly depends on the surface roughness of the substrate and the homogeneity of the laser beam.
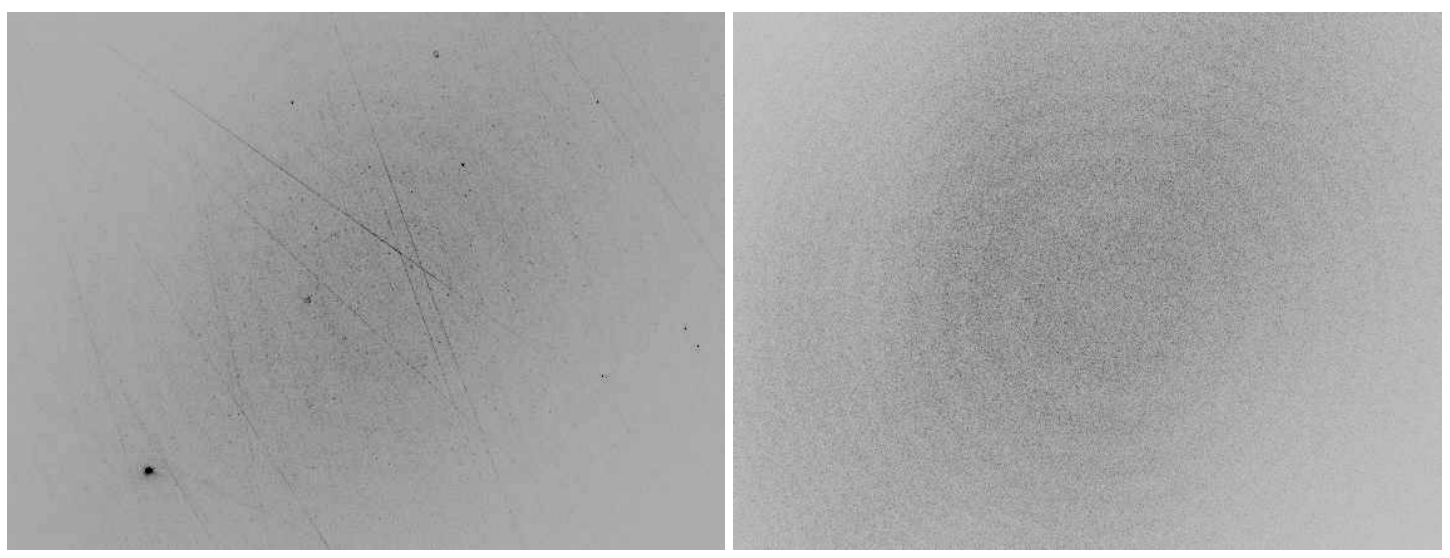

Figure 2: Image (negatives, increased contrast) of particles on a wafer (left) and background of a chromium coated substrate (right)

The negative images shown in Figure 2 are presented with an increased contrast to show the variation in background. Not only scratches but also the beam profile of the laser shows up. The background intensity of images from a chromium coated substrates were about a factor 20 higher than for the silicon process wafer. This difference is most probably due to a higher surface roughness. A reticle substrate coated with Chromium Nitride gave comparable results to the chromium coated blank. Therefore, the image should be corrected for background and beam shape effects for reliable data processing.

Peak levels of a particle on several locations within the image were determined. From the same set of images the average background grey values on particle locations were obtained. The peak levels of the particle signal were linearly dependent on the background level (see Figure 3).

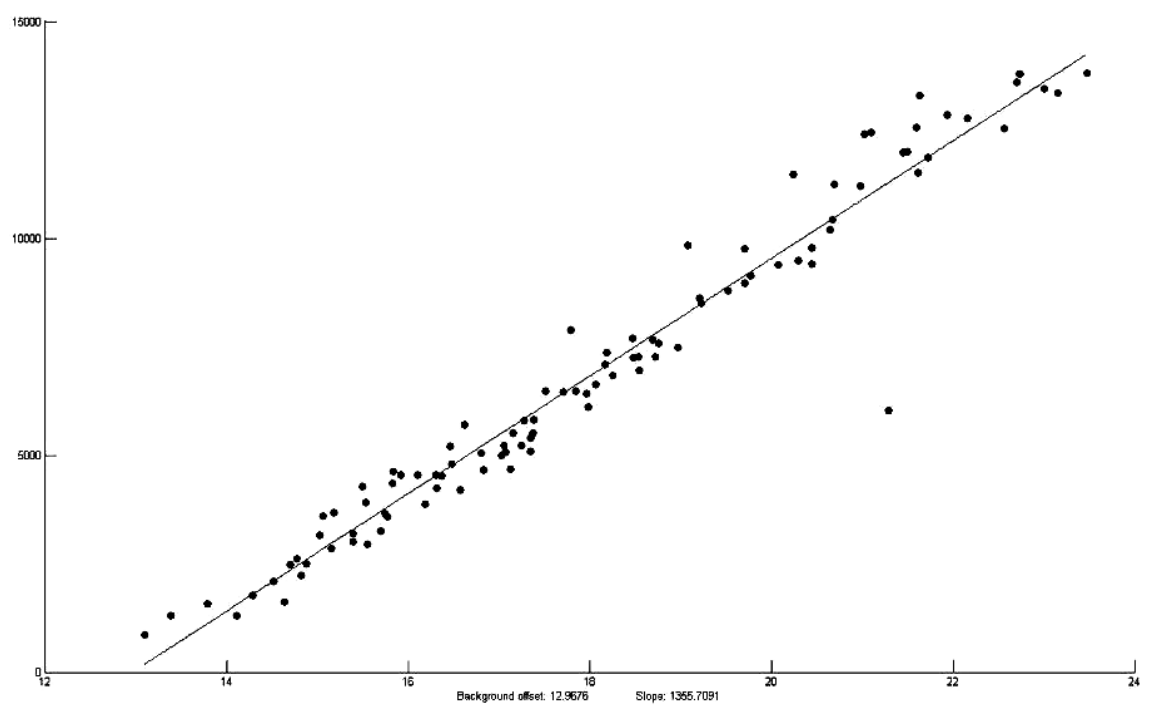

Figure 3: Particle signal versus background signal. 
The linear behavior as shown in Figure 3 enables a correction by calculating the normalized intensity $\Delta$ :

$$
\Delta=\left|\frac{x-\bar{x}-x_{d}}{\bar{x}}\right|
$$

In this equation, $\mathrm{x}$ is the grey value of the pixel, $\bar{x}$ is the average grey value around the particle and $\mathrm{x}_{\mathrm{d}}$ is a camera offset.
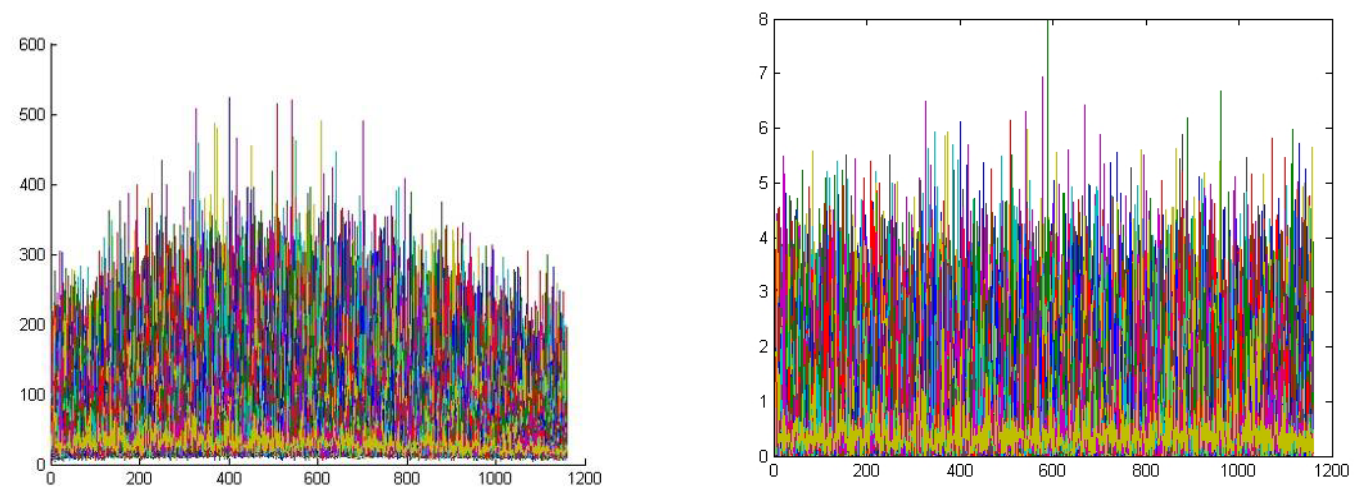

Figure 4: grey values of an image from a chromium substrate as a function of the pixel coordinate in vertical direction; raw image left), normalized to the background level (right)

In Figure 4 (left) the grey values over all vertical lines are given. A large variation in the grey values of the background was observed. Two contributions can be distinguished: a noise type variation and a location dependent variation. The noise is due to a speckle like interference phenomenon. The location dependent variation is caused by the beam profile. In the image centre a higher laser intensity is present than on the sides. By normalizing the pixel grey values to the average local background grey value the intensity differences of the beam shape are corrected for (see Figure 4).

Based on the variations a criterion for the presence of particles can be deducted: if the normalized pixel intensity $\Delta$ surpasses a threshold value, it is counted as a particle. From Figure 4 (right), it can be deducted that a threshold value of 10 will be sufficient to suppress false positives. Figure 5 (left image) shows the same wafer sample as illustrated in Figure 2. In the right image the particle positions (as present on the wafer) are illustrated (determined by the $\Delta>10$ criterion). By using this criterion, scratches were filtered out. Some smaller particles did not exceed the threshold and were rejected. The threshold value determines the size limit for detection and the ratio of false positives and false negatives.
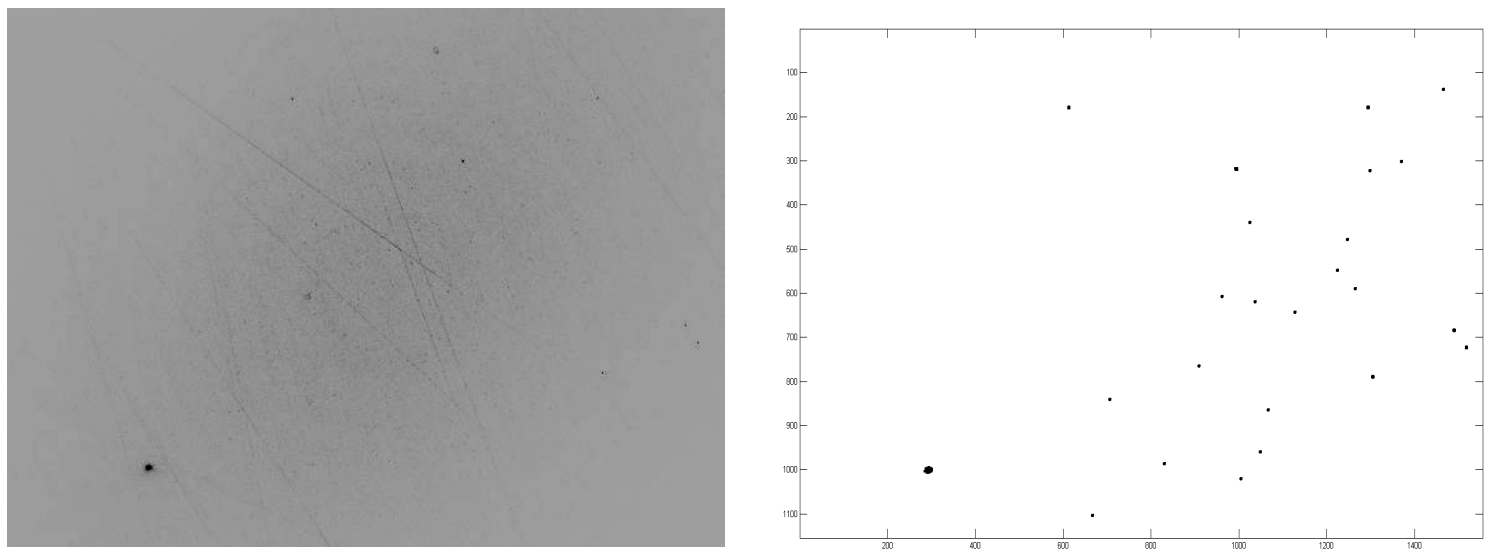

Figure 5: Wafer dark field image(left) and detected particles using $\Delta>10$ criterion (right)

To determine the signal to noise ratio of different particle sizes, a silicon wafer ( $\mathrm{Ra}=0.4 \mathrm{~nm}$ RMS) containing PSL particles $(30 \mathrm{~nm}, 50 \mathrm{~nm}$ and $80 \mathrm{~nm}$ ) was measured with the Rapid Nano. After processing the data grey value cross 
sections were depicted from pixel rows containing a PSL particle (see Figure 6). The background level was nearly similar to the camera offset and the noise level can be calculated from the variations in pixel grey value. For $80 \mathrm{~nm}$ and $50 \mathrm{~nm}$ PSL particles, signal to noise ratios larger than 25 were observed. With these signal to noise ratios particles can be easily detected. The measured signal to noise ration for $30 \mathrm{~nm}$ PSL particles is low. With the used equipment settings 30 nm PSL particles cannot be detected with high reliability.

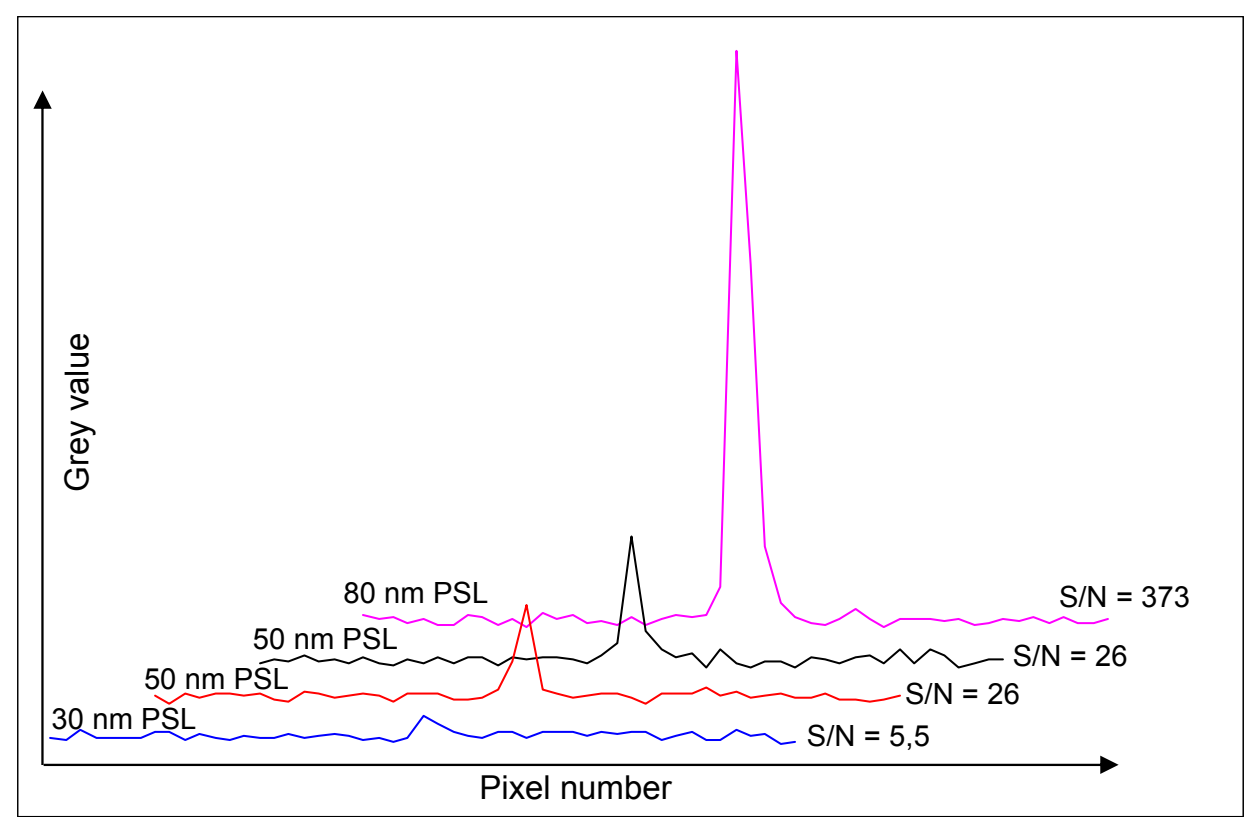

Figure 6: Peak values for PSL particles $(30 \mathrm{~nm}, 50 \mathrm{~nm}$ and $80 \mathrm{~nm})$ and signal noise ratio

The ITRS roadmap ${ }^{[1]}$ shows that relevant particle sizes for EUV reticles are $20 \mathrm{~nm}$ and larger. It is shown that the Rapid Nano is capable of detecting particles of $50 \mathrm{~nm}$ and larger on silicon substrates. The detection limit on other substrates depends on the surface roughness of the substrate. On substrates with a high surface roughness, like chromium or Chromium Nitride coated substrates, we are capable of detecting particles larger than $100 \mathrm{~nm}$. MoSi reticle substrates have a much lower surface roughness. For this type of substrate we expect that the signal to noise ratios for particles smaller than $50 \mathrm{~nm}$ can be increased above to the threshold level.

For the measurement of reticle sized substrates a series of images are made in a stepping mode. After saving an image, the reticle is moved over 1 field of view before the next image is acquired. For a full reticle about 18000 steps are required. The Rapid Nano is capable is performing this task in 2.5 hours. All images are arranged in a mosaic screen and the small overlapping areas are stitched with pixel precision level (see Figure 7). 


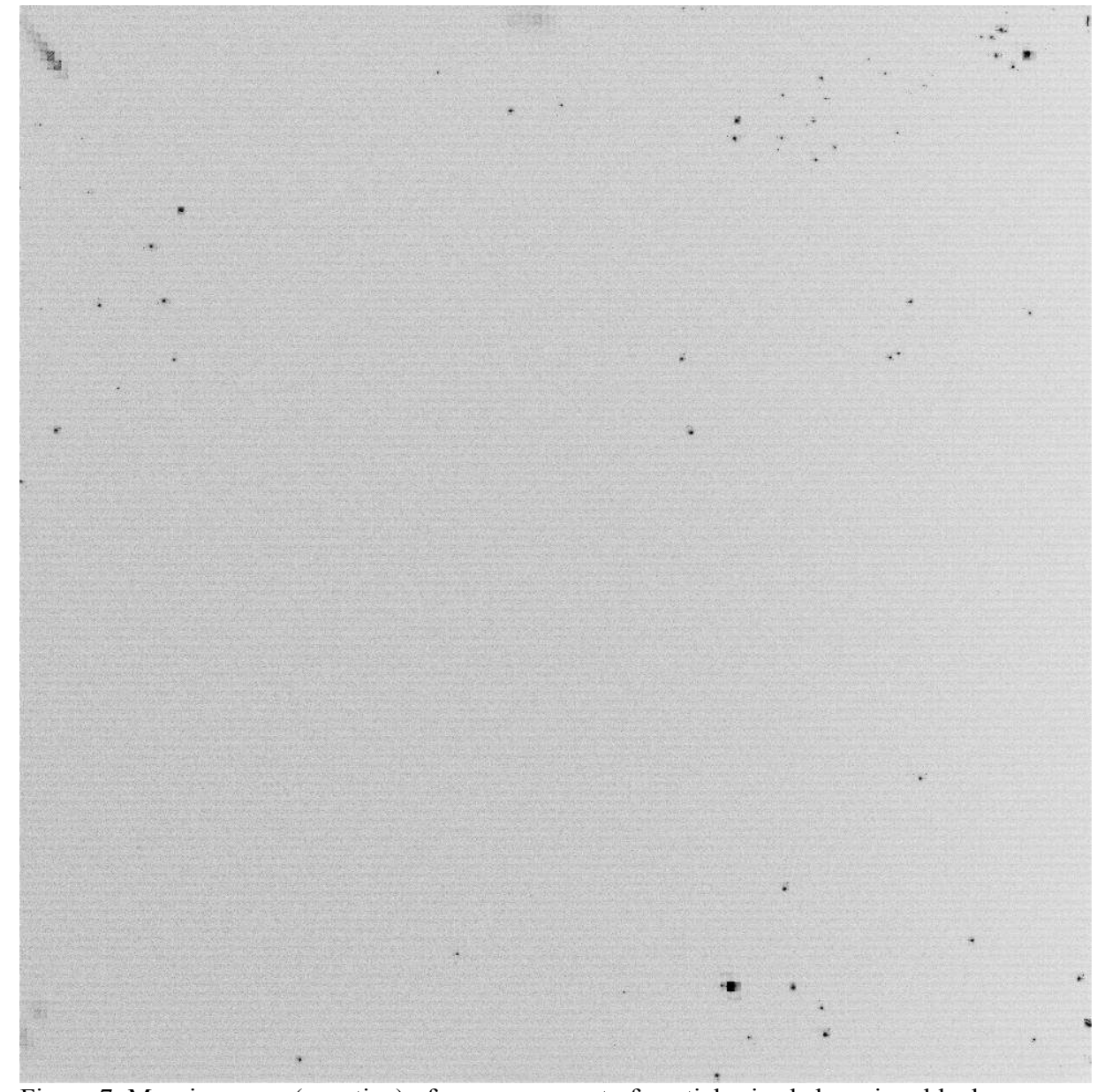

Figure 7: Mosaic screen (negative) of a measurement of a reticle sized chromium blank

After measuring the complete substrate, the data are processed. Particle location and sizes are determined and tabulated in a KLARF-format. When two subsequent measurements are carried out, the particle lists can be cross-linked. In the cross linking procedure all particles present in both scans, added particles and disappearing particles are determined. In an overlay, the locations of all detected particle are indicated and review of the raw data is possible.
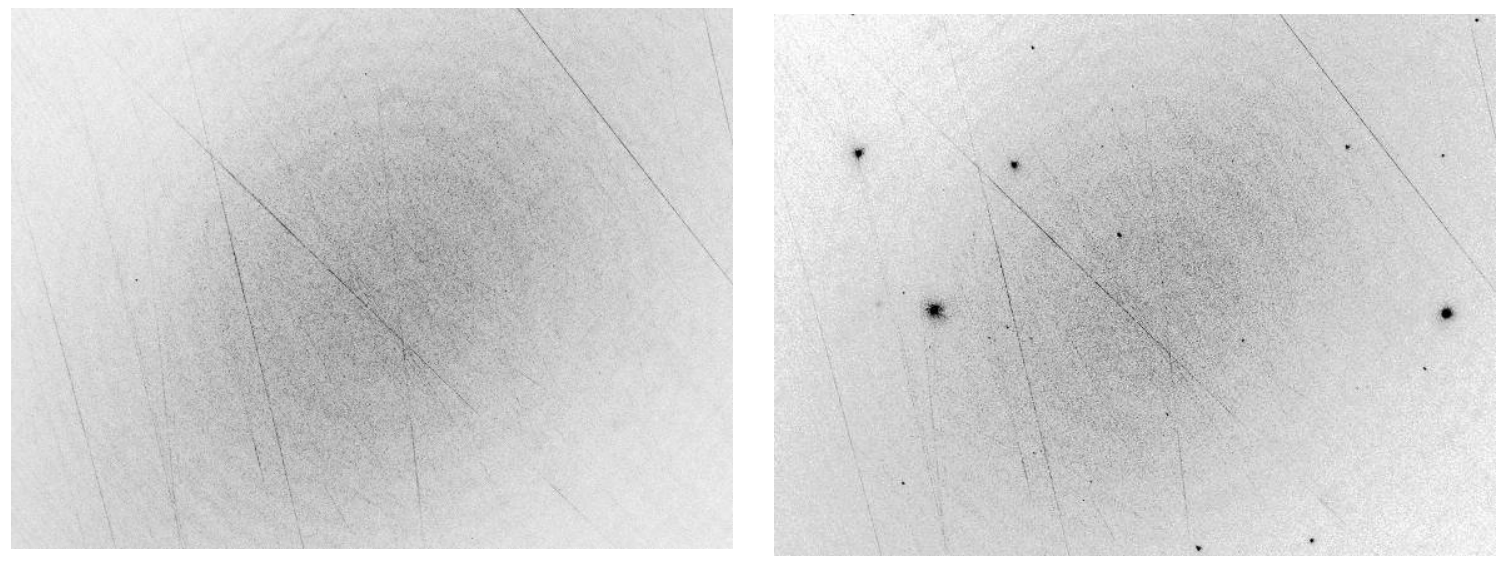

Figure 8: Review images (negatives, increased contrast) of particles on a silicon process wafer, initial measurement (left) after exposure to office air (right). 
Figure 8 gives an example of the review capabilities. A surface area (silicon process wafer $(100 \mathrm{~mm}, \mathrm{Ra}=0.2 \mathrm{~nm}$ RMS)) of 20 x 20 images was measured by the Rapid Nano. After 1 hour exposure to office air the wafer was measured again. In Figure 8 left a review image from the initial measurement is given. Figure 8, right shows a review image of the same location from the second measurement. A large number of detectable added particles is present. The position of the scratches in both images shows the location accuracy of the Rapid Nano.

The applicability of the review capability will vary upon its use. When the Rapid Nano is used for quality control in substrate production, throughput will be very important. For equipment development and qualification purposes reliability and review capabilities will dominate in importance. In these applications it is expected that the number of added particles is relatively low. Therefore, the number of reviews will be low as well.

\section{CONCLUSIONS}

EUV reticles are much more vulnerable to particles than $193 \mathrm{~nm}$ reticles. Therefore, equipment for the handling chain of EUV reticles must meet much more severe requirements on cleanliness than before. The development of new EUV compatible equipment can benefit from the presence of particle inspection equipment. Furthermore, it is expected that each tool which handles EUV reticles must be fully qualified on cleanliness before it may be used. To reach and qualify these cleanliness levels measurement equipment and qualification procedures are a necessity. Because of this requirement, the need for a low cost particle inspection tool is expected.

Rapid Nano; The TNO Particle Scanner, is a dark field imaging based inspection tool for the detection of nano-particles on reticle sized substrates. The Rapid Nano is currently capable of detecting $50 \mathrm{~nm}$ PSL particles with a signal-noise ratio of 26. The signal noise ratio increased with particle size. By continuously moving the substrate over 1 field of view the whole substrate can be measured in 2.5 hours. The Rapid Nano is capable of comparing two measurements. The availability of raw images enables direct defect review, reprocessing at different sensitivity levels and comparison between different measurements. This procedure enables the detection of a single added particle of $50 \mathrm{~nm}$ or larger on a reticle sized substrate. These properties make the Rapid Nano suitable for development support and qualification measurements.

\section{REFERENCES}

[1] ITRS Roadmap 2009.

[2] Meiling, H., Buzing., N., Cummings, K., Harned, N., Hultermans., B., Jonge, R .de, Kessels, B.,Kürz, P., Lok., S., Lowisch., M., Mallman., J., Pierson., B., Wagner., C., Dijk., A.van, Setten., E. van, and Zimmerman J., "EUVL System - Moving Towards Production", Proc. Of SPIE Vol. 7271, 727102, 2009.

[3]Stortelder, J.K., Donck, J.C.J. van der, Oostrom, S., Walle, P. van der, Dress, P., and Brux, O., "Particle qualification procedure for the TNO EUV reticle load port module of the HamaTech MaskTrackPro cleaning tool", Porc. Of SPIE Advanced Lithography 2011.

[4] Peters, J. H., Tonk, C. , Spriegel, D., Han, H.-S., Cho, W. , and Wurm, S., "EUV blank inspection”, EMLC 2008, January $21-24,2008$, Dresden, Germany.

[5] S. Stokowski and M. Vaez-Iravani, Wafer inspection technology challenges for ULSI manufacturing. Proc. Int. Conf. Characterization Metrol. ULSI Technol. 449 (1998), pp. 405-415.

[6] Heerens, G.-J., "Container for a mask", EP1434094A1. 